\title{
Towards a Full Quantum Theory of Black Holes
}

\author{
Claus Kiefer \\ Fakultät für Physik, Universität Freiburg, Hermann-Herder-Straße 3, D-79104 Freiburg, \\ Germany
}

\begin{abstract}
This review gives an introduction to various attempts to understand the quantum nature of black holes. The first part focuses on thermodynamics of black holes, Hawking radiation, and the interpretation of entropy. The second part is devoted to the detailed treatment of black holes within canonical quantum gravity. The last part adds a brief discussion of black holes in string theory and quantum cosmology.
\end{abstract}

Report Freiburg THEP-97/24; to appear in Black Holes: Theory and Observation, edited by F.W. Hehl, C. Kiefer, and R. Metzler (Springer, Berlin, 1998).

\section{Introduction and Summary}

It is of fundamental importance to obtain a full quantum description of black holes. The reasons are of a technical, conceptual, and observational nature. Technical, because it provides a highly nontrivial application of quantum gravitational equations in the full, non-perturbative, regime. One of the main open issues thereby is what substitutes the classical singularities in quantum theory. Conceptual, because the present status of semiclassical approaches leads to problems such as the information loss problem, which can be satisfactorily dealt with only in the full theory. Observational, because apart from potential cosmological data this is probably the only window to directly test a quantum theory of gravity.

This goal has not yet been reached, since a consistent theory of quantum gravity has not yet been constructed. Many quantum aspects of black holes, however, have been understood in the last 25 years, which could lead the way to a full understanding. This review article is intended to give a pedagogical introduction to results which have been obtained in the framework of present approaches towards quantum gravity.

In Sect. 2, I shall review the key issues which lead to the conclusion that black holes are quantum objects. The issues are thermodynamics of black holes, Hawking radiation, and the interpretation of black hole entropy. Since many of these topics are discussed at great length by other lecturers, in particular by 't Hooft, Israel, Neugebauer, and Wipf, I shall present only those issues which I consider to be of particular relevance.

Sect. 3 presents one approach towards a theory of quantum gravity in some detail - the canonical quantisation of general relativity. This approach by itself most likely leads to an effective theory only, but it is the most straightforward 
approach available and offers by itself interesting insights into possible quantum aspects of black holes. The issues addressed cover both applications of the "full" theory (such as a wave function for the eternal Reissner-Nordström hole) and the semiclassical expansion (such as the description of Hawking radiation and black hole entropy in the context of the Wheeler-DeWitt equation).

Sect. 4, finally, gives a brief introduction to superstrings and the issue of black hole entropy as being obtained from counting the states of D-branes. I shall also offer some speculations about the role of black holes in quantum cosmology.

\section{Why Black Holes are Quantum Objects}

\subsection{Thermodynamics of Black Holes}

In the beginning of the seventies, a surprising analogy was discovered between black holes and thermodynamical systems in the framework of general relativity, see the lectures by Israel and Neugebauer in this volume. (Other reviews are, e.g., Bekenstein (1980), Wald (1994, 1997a), and Kiefer (1997a)). This analogy is summarised in Table 1 (with an obvious notation):

Table 1. The laws of black hole mechanics

\begin{tabular}{l|l|l} 
Law & Thermodynamics & Stationary Black Hole \\
\hline Zeroth & $\begin{array}{l}T \text { constant on a body } \\
\text { in thermal equilibrium }\end{array}$ & $\begin{array}{l}\text { surface gravity } \kappa \text { constant on the } \\
\text { horizon of a black hole }\end{array}$ \\
First & $\mathrm{d} E=T \mathrm{~d} S-p \mathrm{~d} V+\mu d N$ & $\mathrm{~d}\left(m c^{2}\right)=\frac{\kappa c^{2}}{8 \pi G} \mathrm{~d} A+\Omega \mathrm{d} J-\phi \mathrm{d} q$ \\
Second & $\mathrm{d} S \geq 0$ & $\mathrm{~d} A \geq 0$ \\
Third & $T=0$ cannot be reached & $\kappa=0$ cannot be reached
\end{tabular}

In the following I shall mostly deal with nonrotating holes $(J=0)$, but often keep a nonvanishing charge $q$. This is not realistic from an astrophysical point of view, but provides an interesting nontrivial example which mimics in many examples the relevant case of rotating holes.

Some comments are appropriate for the Third Law, because this will also be relevant for Sect. 3. In ordinary thermodynamics, there exist various inequivalent formulations of this law. One version frequently used was introduced by Planck in 1911: The entropy $S$ goes to zero (or a material-dependent constant) as the temperature $T$ goes to zero. From this (and some mild assumptions) follows a 
weaker version: $T=0$ cannot be reached in a finite number of steps, see e.g. Wilks (1961) for details. It is this version of the Third Law that was proven by Israel (1986) for black holes and that is stated in Table 1. (In the proof the validity of the weak energy condition for matter in a neighbourhood of the apparent horizon was used.)

$S \rightarrow 0$ as $T \rightarrow 0$ is very helpful in thermodynamics, since it allows one to determine the entropy from measurements of specific heats, $C$. It follows from Planck's version of the Third Law that $C \rightarrow 0$ as $T \rightarrow 0$, but not vice versa (as is sometimes erroneously stated). Planck's version is not always fulfilled; it is violated, for example, for glasses (which have a higher disorder than the corresponding cristalline state). Other examples include the molecule $\mathrm{CH}_{3} \mathrm{D}$ (Straumann 1986) or a gas confined to a circular string at zero temperature (Wald 1997b). From the point of view of quantum statistics it is clear that Planck's version holds if there is a unique non-degenerate ground state at $T=0$. This is violated in these examples.

The above analogy between black hole mechanics and ordinary thermodynamics holds in a much more general framework than general relativity, see Iyer and Wald (1994, 1995), and Wald (1997a). If one only assumes that the field equations follow from a diffeomorphism covariant Lagrangian, $L$, the First Law holds (whether a generalisation of the area theorem holds is not clear).

The term ${ }^{1} \kappa \mathrm{d} A / 8 \pi G$ occurring in the First Law is replaced by

$$
\mathrm{d} \int_{\mathcal{C}} \mathrm{Q}=\frac{\kappa}{2 \pi} \mathrm{d} \mathcal{S}, \quad \text { with } \mathcal{S} \equiv-2 \pi \int_{\mathcal{C}} \frac{\delta L}{\delta R_{a b c d}} n_{a b} n_{c d}
$$

where $\mathrm{Q}$ is the Noether charge 2-form associated with the Killing field $\xi$ normal to the horizon, where the presence of a bifurcate Killing horizon is assumed $(\mathcal{C}$ is the bifurcation surface); $n_{a b}$ denotes the binormal to $\mathcal{C}\left(\nabla_{a} \xi_{b}=\kappa n_{a b}\right)$. For the special case of general relativity, $L=R \sqrt{-g} / 16 \pi G$, the corresponding expression in Table 1 is recovered. If one, on the other hand, assumes beforehand that $S \propto A$, the Einstein field equations must hold (Jacobson 1995).

For generalisations of the laws of black hole mechanics to cases where nonabelian matter fields are present I refer to Heusler (1996), and the references therein.

For completeness I want to mention another, different, analogy between black holes and statistical mechanics: Choptuik (1993) discovered through numerical studies that if a spherical wave packet of a massless scalar field collapses, there exists a critical parameter (characterising the strength of the ensuing gravitational self-interaction of the field) above which no black hole forms. In the vicinity of this critical parameter there is a universal relation for the black hole mass like in the vicinity of a critical point in statistical mechanics.

\subsection{Hawking Radiation}

The analogies between ordinary thermodynamics and black hole mechanics, summarised in Table 1, were first regarded as purely formal, since classically a black

\footnotetext{
${ }^{1}$ From now on we set $c=1$.
} 
hole cannot radiate (it behaves like an ideal absorber). Can quantum theory change this conclusion? One could imagine that $T_{B H} \propto \hbar$ and $S_{B H} \propto \hbar^{-1}$; in fact, from dimensional arguments one recognises that to achieve $T_{B H} \neq 0$ one would have $T_{B H} \propto \hbar \kappa / k_{B}$ and $S_{B H} \propto k_{B} A / G \hbar$, since no other fundamental constants are at one's disposal (at least within standard physics).

Using quantum field theory on a curved background spacetime, Hawking (1975) was able to show that black holes do in fact radiate and have a finite entropy. The temperature is

$$
T_{B H}=\frac{\hbar \kappa}{2 \pi k_{B}},
$$

and the entropy therefore from the First Law

$$
S_{B H}=\frac{k_{B} A}{4 G \hbar} .
$$

This is a very general result, since no use of particular gravitational field equations was made.

This is the reason why black hole thermodynamics seems to hold in a much wider framework, see (1). One there has the formal expression $S_{B H}=k_{B} \mathcal{S} / \hbar$, which would thus give a general local geometric notion of black hole entropy. However, no quantum field theoretical calculation has been made to justify this interpretation.

For later convenience I give the explicit expressions for a Reissner-Nordström black hole (a charged spherically symmetric black hole),

$$
T_{B H}=\frac{\hbar}{8 \pi k_{B} G m}\left(1-\frac{q^{4}}{R_{0}^{4}}\right),
$$

where

$$
R_{0}=G m+\sqrt{(G m)^{2}-q^{2}}
$$

is the radius of the event horizon. The entropy is

$$
S_{B H}=\frac{k_{B}}{G \hbar} \pi R_{0}^{2} .
$$

An extremal hole is defined by $|q|=G m$; its temperature thus vanishes, while its entropy is nonvanishing (and not a constant). It thus seems as if Planck's version of the Third Law were violated, but the situation for extremal holes is more subtle, as will be discussed in Sect. 3. Holes with $|q|>G m$ exhibit a naked singularity and are therefore generally excluded from consideration, although their role within quantum gravity is unclear.

How can one interpret Hawking radiation? The central point is that the notion of vacuum (and therefore also the notion of particles) loses its invariant meaning in the presence of a dynamical background. Incoming modes of the quantum field are redshifted while propagating through the collapsing geometry, which is why the quantum state of the outgoing modes is different. If the initial 
state is a vacuum state, the outgoing state contains "particles". The redshift is especially high near the horizon, where the modes spend a long time before escaping to infinity. This is the reason why Hawking radiation is present very long after the collapse is finished for a comoving observer, contrary to what one would naively expect. The presence of the horizon is also responsible for the thermal nature of the radiation, since no particular information about the details of the collapse can enter. It turns out that the vacuum expectation value of the energy-momentum tensor of the quantum field is negative near the horizon, corresponding to a flux of negative energy into the hole (this is the basis for the pictorial interpretation of the Hawking effect, where one partner of a pair of virtual particles can fall into the hole, thus enabling the other partner to become real and escape to infinity, where it can be observed as Hawking radiation). For details of this scenario, I refer to e.g. Wipf (this volume), 't Hooft (1996, and this volume), Birrell and Davies (1982), Wald (1994), and the references therein. The negativity of this expectation value is, like the Casimir effect, a genuine quantum feature.

This negative energy flux leads to a decrease of the black hole mass and is equal to the positive flux of the Hawking radiation at infinity. From a simple application of Stefan-Boltzmann's law, one can heuristically estimate that the time $t\left(m_{P}\right)$ for the hole to lower its mass to roughly the Planck mass $m_{P} \equiv$ $\sqrt{\hbar / G}$ is $t\left(m_{P}\right) \propto m_{0}^{3}$, where $m_{0}$ is the initial mass of the hole. After this stage is reached, the semiclassical calculations used by Hawking (1975) are expected to break down. It is one of the most interesting open features of a full quantum gravity to provide a detailed understanding of this final phase.

How can one observe the Hawking effect? It is easy to estimate that for an initial mass of about one solar mass, $m_{0} \approx m_{\odot}, t\left(m_{P}\right) \approx 10^{65} \mathrm{yrs}$, which is much longer than the age of the Universe. Before this time the radiation is much too weak to be noticeable. The effect can thus not be observed for black holes originating from stellar collapse. Only if primordial black holes were left over from the Big Bang, would there be a hope of observation (if the initial mass of the hole is $m_{0} \approx 10^{15} \mathrm{~g},{ }^{2}$ the final stages of the primordial hole would occur "today"). The amount of primordial holes is strongly constrained by the smoothness of the Big Bang, see Sect. 4. It is thus not clear whether this effect is observable at all. Bousso and Hawking (1997) have investigated pair creation of black holes during an inflationary phase in the early Universe. By applying the no-boundary proposal of Hartle and Hawking (1983), they estimated that no significant number of neutral holes having sufficient initial mass survive inflation.

If "hot" black holes were around, they would contribute to the observed $\gamma$-ray background. Before the final evaporation (about which nothing is known), the spectrum should according to (2) be thermal. Since this is not true for the $\gamma$-ray background, one finds from observations that the number of primordial holes must be less than about $10^{4}$ per pc ${ }^{3}$ (Page and Hawking 1976). Wright (1996) estimated from the anisotropy component of the $\gamma$-ray background in the halo of the Milky Way an upper limit of 0.4 explosions of primordial holes per $\mathrm{pc}^{3}$ and year.

It may also be possible that the existence of primordial black holes can be inferred

${ }^{2}$ The size of such a hole would be only about $10^{-13} \mathrm{~cm}$ ! 
from the variation of quasar luminosities (Hawkins 1993), although this is at present a contentious issue.

It must be mentioned that there exists an effect analogous to the Hawking effect in Minkowski space, discovered by Unruh (1976). An observer with uniform acceleration $a$ observes thermal radiation in the Minkowski vacuum state with a temperature

$$
T_{U}=\frac{\hbar a}{2 \pi k_{B}} .
$$

The common feature with the black hole case is the presence of a horizon which in particular is responsible for the thermal nature of the radiation. In fact, (7) directly follows from (2) upon replacing the surface gravity $\kappa$ by $a$. Israel (1976) showed that observers whose observations are limited by a horizon see a "thermal vacuum state". This follows after summing over the unobservable states behind the horizon. It must be emphasised that near the horizon the black hole geometry resembles the geometry of Rindler spacetime ('t Hooft 1996), which is the spacetime appropriate for an accelerated observer.

For a quasistationary observer near a black hole (i.e., at a fixed radial distance $r$ from the hole), Hawking effect and Unruh effect are intertwined through the formula

$$
T_{B H}(r)=\frac{\hbar \kappa}{2 \pi k_{B} \chi(r)},
$$

where $\chi(r)$ is the redshift factor of the black hole geometry, and the sphericallysymmetric case was assumed. (A position-dependent temperature is a typical feature of gravitational systems.) In the limit $r \rightarrow \infty$ the Hawking effect (2) is recovered (thermal radiation at infinity), while for $r \rightarrow R_{0}$, the effect is purely one of acceleration and (7) is recovered. This "thermal atmosphere" near the horizon plays an important role in many discussions of black hole entropy, see below.

An interesting connection between the Unruh effect and the Schwinger effect (pair creation of charged particles in an external electric field) was discussed by Parentani and Massar (1997). This analogy enabled them to associate a formal entropy with the Unruh effect, $S_{U}=k_{B} \pi M^{2} / e \mathcal{E} \hbar$, where $\mathcal{E}$ is the constant accelerating electric field, and $M$ is the mass of the charged particle. With $a=e \mathcal{E} / M$ one has $S_{U} \propto M^{2}$ and $T_{U} \propto M^{-1}$, i.e. a formal analogy to the Hawking effect (although with a different interpretation, since here $M$ refers to the quantum field, while in the black hole case, $m$ refers to the classical black hole mass).

Can the Unruh effect (7) be observed? Bell and Leinaas (1987) discussed the motion of electrons in storage rings. For such circular motion, the effect is not purely thermal, since there is no horizon. Still, this effect leads to a change in the spin polarisation of the electron, which may be obervable. However, present measurements of this polarisation are not precise enough to unambigiously uncover such an effect from the data.

A related effect (quantum radiation by moving interfaces between different dielectrics) could be responsible for sonoluminescence (light emission by sound-driven air bubbles in water), which until now remains unexplained, see Eberlein (1996). This is undecided at the moment. 


\subsection{Interpretation of Entropy}

If black holes can be attributed a genuine entropy, see (3), the question arises whether a generalised Second Law of the kind

$$
\frac{\mathrm{d}}{\mathrm{d} t}\left(S_{B H}+S_{M}\right) \geq 0
$$

holds, where $S_{M}$ denotes the entropy of ordinary matter. This was investigated in many special situations, and numerous gedankenexperimente have shown that (9) in fact holds, i.e. that there exists no perpetuum mobile of the second kind in black hole physics. A typical situation is one where a box containing thermal radiation (this maximises the matter entropy) is lowered in a quasistationary manner towards a black hole, into which the radiation is then thrown, see Bekenstein (1980), and Israel (this volume). Unruh and Wald (1982) have shown that there is a minimal change of entropy if the box is opened at the floating point given by the Archimedean principle (weight of box is equal to the buoyancy from the Unruh radiation), which is just enough to save the Second Law (9). In this discussion the relation (8) plays an important role.

Frolov and Page (1993) have given a proof for the generalised Second Law (9) under the assumptions that one remains within the semiclassical approximation and that a special initial state (no correlation between modes coming out of the past horizon and modes coming in from past null infinity) is chosen. The choice of a special initial state is of course a necessary prerequisite for any derivation of a Second Law, see Zeh (1992) and Sect. 4.

The above discussion remains fully within the context of phenomenological thermodynamics (similar to discussions in the last century before the advent of the molecular hypothesis). A most interesting question is then whether $S_{B H}$ can be derived from quantum statistical considerations,

$$
S_{B H} \stackrel{?}{=}-k_{B} \operatorname{Tr}(\rho \ln \rho) \equiv S_{S M}
$$

with an appropriate density matrix $\rho$. This is a key issue in the process of understanding black holes in quantum gravity. Does black hole entropy, for example, correspond to the large number of states which may be hidden behind the horizon? Or does it correspond to the large number of possible initial states? Where is the entropy located (if at all)? These question may indicate the kind of questions that arise.

Using a flat space example (with a surface that separates two regions and that mimics a horizon), Bombelli et al. (1986), and Srednicki (1993) have argued that the entropy is located near the horizon. This may also be suggested by the presence of the thermal atmosphere there, see the discussion after (8). In the black hole context, this was investigated by Frolov and Novikov (1993). They showed that by counting internal degrees of freedom one gets $S_{S M} \propto A$. All these authors found, however, a divergent prefactor. Although lying inside, these degrees of freedom are located mainly in the vicinity of the horizon. An 
attempt to show that (10) can be derived from the number of possible initial configurations of the hole was made by Zurek and Thorne (1985).

A concrete realisation of the ideas of Frolov and Novikov (1993) was done by Barvinsky, Frolov, and Zelnikov (1995). They consider a quantum state for the black hole and make the ansatz that this state is constructed from the no-boundary proposal of Hartle and Hawking (1983). The wave function is defined on three-dimensional geometries and matter fields thereon, see Sect. 3. The three-geometry is taken to be the Einstein-Rosen bridge $\Sigma \equiv \mathbb{R} \times S^{2}{ }^{3}$ The density matrix $\rho_{\text {in }}$ of the black hole is then obtained from this pure state by tracing out all degrees of freedom outside the horizon. For the statistical mechanical entropy this leads to

$$
S_{S M}=-k_{B} \operatorname{Tr}\left(\rho_{i n} \ln \rho_{i n}\right)=k_{B} \frac{A}{360 \pi l^{2}},
$$

where $l$ is a cutoff parameter (proper distance to the horizon). One recognises that one gets a divergent result for $l \rightarrow 0$. (Taking for $l$ the Planck length $l_{P} \equiv \sqrt{G \hbar}$ would yield a result proportional to (3).) It is speculated that a finite result is obtained after the quantum gravitational "uncertainty" of the horizon is taken into account, see also Sect. 4 .

Since

$$
\operatorname{Tr}\left(\rho_{\text {in }} \ln \rho_{\text {in }}\right)=\operatorname{Tr}\left(\rho_{\text {out }} \ln \rho_{\text {out }}\right)
$$

(see e.g. p. 297 in Giulini et al. (1996)), the result $S \propto A$ also follows in approaches where the degrees of freedom lie outside the horizon. An example is the "brick wall model" of 't Hooft (1996), see also his contribution to this volume.

The above result by Barvinsky, Frolov, and Zelnikov (1995) arises entirely from the "one-loop level" of the wave function (that is the level of the WKB prefactor). Usually, however, $S_{B H}$, Eq. (3), is recovered solely from the classical action, which corresponds to the "tree level" of approximation. Since this latter type of derivation plays a crucial role in many discussions, and will in particular be of some relevance in Sect. 3, a brief overview will now be given.

The origin of these discussions goes back to Gibbons and Hawking (1977) who extended the analogy between path integrals and partition sums to gravitational systems. This analogy, on the other hand, was introduced within ordinary statistical mechanics by Feynman and Hibbs (1965).

Consider the partition sum of the canonical ensemble,

$$
\mathrm{e}^{-\beta F} \equiv Z=\operatorname{Tre}^{-\beta \hat{H}},
$$

where $\beta=\left(k_{B} T\right)^{-1}$, and $F$ is the free energy. On the other hand, the quantum mechanical kernel of the evolution operator reads

$$
G\left(x, t ; x^{\prime}, 0\right)=\left\langle x\left|\mathrm{e}^{-\mathrm{i} t \hat{H} / \hbar}\right| x^{\prime}\right\rangle=\int \mathcal{D} x(\tau) \mathrm{e}^{\mathrm{i} S[x(\tau)] / \hbar},
$$

\footnotetext{
${ }^{3}$ It is shown that this state is equal to the so-called Hartle-Hawking vacuum state which is relevant for eternal holes, see Hartle and Hawking (1976). This thus provides an example where both types of "Hartle-Hawking" agree.
} 
where also its expression in terms of path integrals is given (the paths going through $x^{\prime}$ at time 0 and through $x$ at time $t$ ). For simplicity, I have suppressed all indices which may be attached to $x$.

The partition sum $Z$ can be evaluated in this way, if one transforms $t \rightarrow-\mathrm{i} \beta \hbar$ and performs a trace:

$$
Z=\int \mathrm{d} x G(x,-\mathrm{i} \beta \hbar ; x, 0)=\int \mathcal{D} x(\tau) \mathrm{e}^{-I[x(\tau)] / \hbar} .
$$

The paths go now from $x$ at "time" 0 back to $x$ at "time" $\beta \hbar$. ( $I$ denotes the euclidean action.) To express $Z$ in this way is especially suited for perturbation theory, see Feynman and Hibbs (1965). If the Hamiltonian has the standard form

$$
\hat{H}=\frac{\hat{p}^{2}}{2 m}+V(\hat{x}),
$$

one finds in perturbation theory (the "small" parameter being $\beta \hbar$ ) for the free energy the expression (see standard books on statistical mechanics)

$$
F=F_{0}+\frac{\hbar^{2} \beta^{2}}{24 m}\left\langle V^{\prime}(x)^{2}\right\rangle,
$$

where the expectation value is performed with respect to the canonical ensemble. The first term, $F_{0}$, gives the classical value for the free energy ("tree level"). It follows from evaluating the classical action upon classical trajectories. Because the action contains an integration from 0 to $\beta \hbar$, for small $\beta \hbar$ (corresponding to $\hbar \rightarrow 0$ or $T \rightarrow \infty$ ) the result for $F_{0}$ is linear in $\beta$ and independent of $\hbar$. The second term in (16) describes the "one-loop level" of the perturbation. It follows from an evaluation of the quadratic fluctuations around the classical action. (There is no term linear in $\hbar$.)

If $Z$ (or $F$ ) is known, all other thermodynamic quantities (in particular the entropy) can be calculated. The mean value of the Hamiltonian is

$$
\langle\hat{H}\rangle \equiv E=-\frac{\partial \ln Z}{\partial \beta},
$$

the entropy is given by

$$
S=k_{B}(\ln Z+\beta E)=\frac{E-F}{T}=-\frac{\partial F}{\partial T} .
$$

One also has $S \approx k_{B} \ln g(E)$, where $g(E)$ is the number of states in the energy interval given by the mean square deviation of the energy. The specific heats can be inferred from second derivates of the partition sum,

$$
C=k_{B} \beta^{2} \frac{\partial^{2} \ln Z}{\partial \beta^{2}}=k_{B}(\Delta \hat{H})^{2} \beta^{2}=-\beta \frac{\partial S}{\partial \beta} .
$$

Gibbons and Hawking (1977) now used a (formal) quantum gravitational path integral to evaluate the partition sum in the gravitational context, see 
also Hawking (1979) and Hawking and Penrose (1996). In contrast to the above standard context, the euclidean viewpoint is there assumed to be fundamental and not just a convenient rewriting of the original lorentzian theory.

The path integral cannot, of course, be evaluated exactly (and it is unclear, whether it can be rigorously defined in quantum gravity). One can, however, resort to a steepest descent (saddle point) approximation, where only the first (and sometimes the second) contribution is taken into account. The first contribution is just the classical action evaluated for a classical solution of Einstein's equations. The next order takes into account the standard WKB-prefactor.

The euclidean action of vacuum general relativity without cosmological constant reads

$$
I=-\frac{1}{16 \pi G} \int \mathrm{d}^{4} x R \sqrt{g}+\frac{1}{8 \pi G} \int \mathrm{d}^{3} x\left(K-K^{0}\right) \sqrt{h} .
$$

In the volume term, $R$ denotes the four-dimensional Ricci scalar, and $g$ the determinant of the four-dimensional metric. In the boundary term, $K$ denotes the trace of the extrinsic curvature, and $h$ the determinant of the three-dimensional metric. For purposes of regularisation in the asymptotically flat case, the trace of the extrinsic curvature $K^{0}$ of the same boundary embedded in flat space has to be subtracted.

If one considers spherically symmetric uncharged black holes, one has to evaluate (20) for the euclidean Schwarzschild solution (the generalisation to $q \neq 0$ is straightforward). For this solution $R=0$, and there is thus no contribution from the volume term. The whole contribution (which I shall call $I^{*}$ ) thus arises from the boundary which here is the $t$-axis times a sphere of large radius. This is a typical feature of black hole physics, which we shall encounter again in the course of this lecture.

To evaluate the partition sum one has to start from the expression (14), where one has to sum over all four-dimensional metrics instead of just paths $x(\tau)$. In the saddle point approximation one has (denoting with g symbolically the four-dimensional metric),

$$
Z=\int \mathcal{D} g(x) \mathrm{e}^{-I[\mathrm{~g}(x)] / \hbar} \approx \exp \left(-I^{*} / \hbar\right)=\exp \left(-\frac{(\beta \hbar)^{2}}{16 \pi G \hbar}\right)
$$

It is due to the fact that only the boundary term of the euclidean action contributes to (21), that the lowest order approximation of the path integral (the "tree level") depends already quadratically on $\beta$. As one recognises from (16) and the discussion following it, in the standard situation $\beta$ occurs quadratically only at the next order.

From (17) one immediately finds

$$
\langle\hat{H}\rangle=E=\frac{\hbar \beta}{8 \pi G}=m
$$


which leads to the expression (4) for the temperature (with $q=0$ ). From (18) one finds for the entropy

$$
S=k_{B}(\ln Z+\beta m)=\frac{\hbar \beta^{2}}{16 \pi G}=\frac{k_{B} A}{4 G \hbar}=S_{B H} .
$$

If $Z$ had only a linear dependence on $\beta$, the entropy would turn out to be zero. From (19) one finds $C=-\beta^{2} \hbar / 8 \pi G$ and thus a negative specific heat! This is in particular in conflict with the positivity of $(\Delta \hat{H})^{2}$ und means, of course, that the black hole is unstable in asymptotically flat space, as can immediately be inferred from the inverse mass dependence of the Hawking temperature (4). As such, this is not very surprising, since instability is typical for gravitational phenomena (Zeh 1992). This negativity is therefore not an artifact of the treelevel approximation.

Davies (1977) showed that for rotating or charged holes, the specific heat can become positive for $J / m \gtrsim 0.68 \mathrm{Gm}$ (rotating holes, where $J$ is the angular momentum) and $q \gtrsim 0.86 \mathrm{Gm}$ (charged holes).

In the attempt to find a thermodynamically stable situation, Gibbons and Perry (1978) considered a microcanonical ensemble of a black hole immersed in a bath of radiation with fixed volume: They found that at a sufficiently high energy density a black hole will nucleate from a box containing radiation, in the same way as a liquid drop can condense out of saturated vapour. However, to obtain stability the black hole mass $m$ must be about $98 \%$ of the total energy, which means that the radiation cannot serve as a heat bath for the hole.

In a canonical ensemble description, the specific heat can be made positive if the black hole is put into a box (York 1986, 1991). At the boundary of the box, boundary conditions must be specified, i.e. in the Schwarzschild case one can fix the temperature of the box and its radius $r_{B}$. It follows then that stability can be achieved for $2 G m<r_{B}<3 G m$, i.e. only for a very small box.

Alternatively, one can use a microcanonical description, where the energy (and other extensive variables) are fixed at the boundary (Brown and York 1993). This is very natural for gravitating systems where energy can be expressed as a surface integral. Instead of the euclidean path integral (14) for the canonical partition sum, one can express the density of states $\nu(E)$ directly as a lorentzian path integral,

$$
\nu(E)=\int \mathcal{D} x(t) \mathrm{e}^{\mathrm{i} S_{E}[x(t)] / \hbar},
$$

where $S_{E}$ is Jacobi's action in which the energy is fixed. The sum goes over all paths that are periodic in real time. This path integral may be defined even in cases where the canonical partition function (which follows via an integral transform) is divergent. Brown and York (1993) showed that $\ln \nu \approx A / 4 G \hbar$, as long as the black hole can be described semiclassically by any real stationary axisymmetric black hole.

If the hole is charged, one must in addition fix the charge at the boundary or, alternatively, the electric potential, see Braden et al. (1990). 
Iyer and Wald (1995) gave a comparison between the Noether charge approach, see (1), and various euclidean approaches. They showed that the results agree in their respective domains of applicability, see also Brown (1995). It is interesting that $\exp \left(S_{B H}\right)$ also gives the enhancement factor for the rate of black hole pair creation relative to ordinary pair creation, in accordance with the heuristic interpretation of this factor as the number of internal states of the hole.

Can these derivations of black hole entropy at the tree level be reconciled with the above-mentioned derivations at the one-loop level, see (11)? Problems arise due to the UV-divergencies connected with one-loop calculations: For renormalisation one needs to subtract the infinite quantity $S_{B H}\left(G_{b a r e}\right)$ evaluated at the "bare" gravitational constant $G_{b a r e}$, a quantity that has no clear statistical mechanical meaning. As Frolov, Fursaev, and Zelnikov (1997) have shown, this difficulty can be avoided in theories where $G_{\text {bare }}^{-1}=0$, such as Sakharov's induced gravity, see also Frolov and Fursaev (1998) for a review: If one includes there non-minimally coupled scalar fields or additional vector fields, one obtains a finite entropy that is equal to $S_{B H}$. In induced gravity, the dynamical degrees of freedom of the gravitational field arise from collective quantum excitations of heavy matter fields. The same fields produce $S_{B H}$, since the gravitational action is already itself a "one-loop effect". This result may also indicate why superstring theory, another "effective theory of gravity", allows one to reproduce $S_{B H}$ from the counting of quantum states, see Sect. 4.

It was the intention of this section to give convincing arguments that black holes must be quantum objects and that they can be fundamentally understood only in the framework of quantum gravity. Before I shall discuss some approaches to quantum gravity in more detail, I want to remark that one can already speculate from the above results about some possible features of the full theory. One result of such a speculation is the intriguing feature of a possible area (and thus mass) quantisation for a black hole, see e.g. Bekenstein (1997), and the references therein. It was suggested from heuristic considerations that

$$
A=16 \pi(G m)^{2}=4 G(\ln 2) \hbar n, \quad n \in \mathbb{N} .
$$

This would already in the semiclassical theory change drastically the spectrum of black hole radiation. For example, no quanta would be emitted with frequencies lower than some fundamental frequency $(\ln 2) / 8 \pi G m$, in contrast to the thermal nature of Hawking radiation. One could thus test this effect of quantum gravity already for $m \gg m_{P}$ (provided that primordial holes exist).

The result (2) of a thermal spectrum of black hole radiation was obtained in the semiclassical limit, where gravity is treated classically. If it were true even in the full theory of quantum gravity, it would mean that "information" were lost in the following sense: Since one can in principle start from any initial quantum state (even a pure one), its exact evolution into a thermal state would contradict the unitary evolution law of standard quantum theory. In this case, a theory of quantum gravity would possess some radical new features. Since, however, the full theory is not yet known, the answer to this problem of information loss is also not yet known (see, for example, the review in Giddings 1994). This "problem" 
may, however, serve as a useful leitmotif in the search for a full theory. How even the semiclassical limit might be altered has been mentioned in the context of (24). The effect of quantum gravitational corrections on this information loss will be briefly discussed in Sect. 3.2.

\section{Black Holes in Canonical Quantum Gravity}

\subsection{A Brief Introduction into Canonical Gravity}

Canonical quantum gravity is obtained via the application of standard canonical quantisation rules to the theory of general relativity (or some other classical theory, but I shall restrict myself to general relativity). Since this does not provide a unified description of all fields, it is expected that the resulting framework is only an effective theory. There is, however, the hope that canonical quantum gravity may reflect many of the features of a genuine quantum theory of gravity. Its formulation must be intrinsically non-perturbative, since general relativity is known to lead to a non-renormalisable quantum theory at the perturbative level. A perhaps more serious candidate for a genuine quantum theory of gravity unifying all interactions, superstring theory, is briefly described in the next section.

The canonical framework assumes that the classical spacetime $\mathcal{M}$ is globally hyperbolic, $\mathcal{M}=\Sigma \times \mathbb{R}$, such that a $3+1$ decomposition (a foliation into spacelike hypersurfaces) can be performed. This is already of relevance for the classical theory because it allows one to pose a well-defined Cauchy problem (e.g. in numerical relativity, see the contribution of Seidel to this volume). A $3+1$ formulation is required because the canonical approach is a Hamiltonian formulation of the theory. Due to the four-dimensional diffeomorphism invariance ("coordinate invariance" in spacetime), the classical theory contains four constraints at each space point, one Hamiltonian constraint,

$$
\mathcal{H} \approx 0,
$$

and three spatial diffeomorphism constraints ("coordinate invariance" on the three-dimensional spatial hypersurface $\Sigma$ ),

$$
\mathcal{D}_{a} \approx 0 \text {. }
$$

Here, as usual, $\approx$ denotes the weak equality in the sense of Dirac.

The canonical configuration variable can be chosen to be the three-dimensional metric $h_{a b}(\mathbf{x})$ on $\Sigma$, and the canonical momentum is then a linear function of the extrinsic curvature of $\Sigma$. To this one can add any matter fields in the standard manner. This constitutes the traditional, geometrodynamic, approach. Alternatively, one may choose a complex connection or so-called loop variables on $\Sigma$ for the configuration variables. This brings in many formal similarities to Yang-Mills theories. I want to emphasise that the constraint structure $(25,26)$ is typical for all versions of canonical theories that possess a diffeomorphism invariance on 
the classical level, even if the specific form is different. This is the basis for the hope that these versions have important common features. Also superstring theory has a constraint structure, although its interpretation is somewhat different from here.

In the following I want to restrict myself to the quantisation method proposed by Dirac. This means to formally transform the above constraint equations into operator equations acting on physical states $\Psi$,

$$
\hat{\mathcal{H}} \Psi=0,
$$

and

$$
\hat{\mathcal{D}}_{a} \Psi=0 .
$$

The wave functional $\Psi$ depends, in the geometrodynamic approach, on the threemetric (as well as on non-gravitational fields), in the other approaches mentioned above on the complex connection or on loop variables. ${ }^{4}$ Due to the constraints (28), the wave functional is invariant under three-dimensional coordinate transformations. This is often indicated by writing $\Psi\left[{ }^{3} \mathcal{G}\right]$, where ${ }^{3} \mathcal{G}$ means "threegeometry", although this is a loose notation, since $\Psi$ cannot explicitly be given in this form.

If space is compact, there are no further constraints. If not, additional constraints arise from variables living at boundaries. This will be of particular relevance for our treatment of black holes, see Sect. 3.2.

It cannot be the purpose of this article to give a detailed introduction into this approach and its problems. A comprehensive reference is Ehlers and Friedrich (1994). A recent report on the connection and loops approaches can be found, for example, in Ashtekar (1997); a recent report on conceptual problems in Isham (1997). A comprehensive review of canonical quantum gravity as applied to cosmology is Halliwell (1991). The black hole examples discussed below may also be thought to give illustrative examples for the full framework.

A helpful analogy between ordinary (quantum) mechanics and (quantum) general relativity is given in Table 2 .

The most important conceptual lesson from the above comparison is that spacetime has no fundamental meaning in canonical quantum gravity, in the same way as a particle trajectory has no fundamental meaning in quantum mechanics. This fact lies behind the so-called "problem of time" in quantum gravity - the absence of any external time parameter in the constraint equations $(27,28)$, and the related problem of which Hilbert space (if any) to choose in quantum gravity. (This is way the quantum gravitational wave function in Table 2 is $t$-independent.) To a large extent, these issues are open, see e.g. Kiefer (1997b). Fortunately, in the black hole case, the "rest of the Universe" can be assumed to be in a semiclassical regime where a concept of time exists, so that some of the above conceptual problems don't have to be dealt with in the first place. These problems are, however, relevant if the whole Universe including the black hole is described in quantum terms, see Sect. 4.

\footnotetext{
${ }^{4}$ In the latter cases there are also additional constraints coming from triad rotations.
} 
Table 2. Comparison of mechanics and general relativity

\begin{tabular}{l|l} 
Mechanics of one particle & General relativity \\
\hline position $q$ & $\begin{array}{l}\text { geometry }{ }^{3} \mathcal{G} \text { of a } \\
\text { three-dimensional space }\end{array}$ \\
trajectory $q(t)$ & spacetime $\left\{{ }^{3} \mathcal{G}(t)\right\} \equiv{ }^{4} \mathcal{G}$ \\
uncertainty between & $\begin{array}{l}\text { uncertainty between } \\
\text { "space and time" } \\
(\text { three-geometry and extrinsic curvature) }\end{array}$ \\
$\psi(q, t)$ & $\Psi\left[{ }^{3} \mathcal{G}, t\right) \equiv \Psi\left[{ }^{3} \mathcal{G}\right]$
\end{tabular}

A frequently employed approximation scheme is to perform a semiclassical expansion of the equations $(27,28)$, see Kiefer (1994). One writes the full wave functional as $\Psi \equiv \exp (\mathrm{i} S / \hbar)$ with some arbitrary complex function $S$ which is expanded into powers of the gravitational constant: $S=G^{-1} S_{0}+S_{1}+G S_{2}+\ldots$. This is then inserted into $(27,28)$, leading to equations at consecutive orders of $G$. It must be emphasised that this can be done only in a formal way, since it is unclear how to rigorously define the equations $(27,28)$. For finite-dimensional models it was shown by Barvinsky and Krykhtin (1993) and Barvinsky (1993) how up to "one loop" a consistent factor ordering and a consistent Hilbert space structure can be obtained. The important open issue is to find a consistent, anomaly-free, regularisation for their equations in the field theoretic case.

The highest order $\left(G^{1}\right)$ yields the gravitational Hamilton-Jacobi equation for $S_{0}$. This is equivalent to the classical Einstein equations and corresponds to the "tree level" of the theory. A special solution $S_{0}$ thus corresponds to a family of classical spacetimes. The next order $\left(G^{0}\right)$ leads to a functional Schrödinger equation for non-gravitational fields in a given background. It corresponds to the "one-loop" limit of quantum field theory in an external background, the limit in which the Hawking radiation is derived. Higher orders in $G$ then lead to genuine quantum gravitational correction terms as well as back reaction terms from the non-gravitational fields onto the semiclassical background.

The approximation scheme sketched above is not unique. Alternative schemes can be found, e.g., in Bertoni, Finelli, and Venturi (1996), and Kim (1997). They differ from the above in the treatment of the back reaction of the non-gravitational fields.

The next section is devoted to the application of canonical methods to a particular situation: spherically symmetric black holes. 


\subsection{Quantisation of spherically symmetric black holes}

The first model which I shall briefly describe is the case of spherically symmetric black holes. I shall begin with the so-called "eternal hole", where only the gravitational degrees of freedom (and, in the Reissner-Nordström case, the electromagnetic field) are taken into account. The more realistic case where additional dynamical fields (such as a scalar field) are present is discussed thereafter.

The eternal Schwarzschild hole was first discussed by Thiemann and Kastrup (1993), Kastrup and Thiemann (1994) within the connection dynamical approach and then by Kuchař (1994) in the geometrodynamical approach, see also Cavaglià, de Alfaro, and Filippov (1996). I shall follow the geometrodynamical approach and generalise it to include the Reissner-Nordström case, see also Louko and Winters-Hilt (1996). "Eternal" refers to the time-symmetric case where both a past and a future horizon are present ("complete Kruskal diagramme"). Such holes cannot result from a collapse. Although thus being unrealistic from an astrophysical point of view, eternal holes provide a useful (and relatively simple) framework for questions of principle.

Starting point is the ADM form for general spherical symmetric metrics on $\mathbb{R} \times \mathbb{R} \times S^{2}:$

$$
\mathrm{d} s^{2}=-N^{2}(r, t) \mathrm{d} t^{2}+\Lambda^{2}(r, t)\left(\mathrm{d} r+N^{r}(r, t) \mathrm{d} t\right)^{2}+R^{2}(r, t) \mathrm{d} \Omega^{2} .
$$

The lapse function $N$ encodes the possibility to perform arbitrary reparametrisations of the time parameter, while the shift function $N^{r}$ is responsible for reparametrisations of the radial coordinate (this is the only freedom in performing spatial coordinate transformations that is left after spherical symmetry has been imposed). The parameter $r$ is only a label for the spatial hypersurfaces; if the hypersurface extends from the left to the right wedge in the Kruskal diagramme, one takes $r \in(-\infty, \infty)$. If the hypersurface originates at the bifurcation point where path and future horizon meet, $r \in(0, \infty)$. If one has in addition a spherically symmetric electromagnetic field, one makes the following ansatz for the one-form potential:

$$
A=\phi(r, t) \mathrm{d} t+\Gamma(r, t) \mathrm{d} r
$$

In the Hamiltonian formulation, $\phi$ as well as $N$ and $N^{r}$ are Lagrange multipliers whose variations yield the constraints of the theory. Variation of the EinsteinHilbert action with respect to $N$ yields the Hamiltonian constraint (25) which for the spherically symmetric model reads

$$
\mathcal{H}=\frac{G}{2} \frac{\Lambda P_{\Lambda}^{2}}{R^{2}}-G \frac{P_{\Lambda} P_{R}}{R}+\frac{\Lambda P_{\Gamma}^{2}}{2 R^{2}}+G^{-1} V_{G} \approx 0,
$$

where the gravitational potential term reads, explicitly,

$$
V_{G}=\frac{R R^{\prime \prime}}{\Lambda}-\frac{R R^{\prime} \Lambda^{\prime}}{\Lambda^{2}}+\frac{R^{\prime 2}}{2 \Lambda}-\frac{\Lambda}{2} .
$$


(A prime denotes differentiation with respect to $r$.) Variation with respect to $N^{r}$ yields one (radial) diffeomorphism constraint (26),

$$
\mathcal{D}_{r}=P_{R} R^{\prime}-\Lambda P_{\Lambda}^{\prime} \approx 0
$$

One recognises from this constraint that $R$ transforms as a scalar, while $\Lambda$ transforms as a scalar density.

Variation of the action with respect to $\phi$ yields as usual the Gauß constraint

$$
\mathcal{G}=P_{\Gamma}^{\prime} \approx 0
$$

The constraint (33) generates radial diffeomorphisms for the fields $R, \Lambda$ and their canonical momenta. It does not generate diffeomorphisms for the electromagnetic variables. This can be taken into account if one uses the multiplier $\tilde{\phi}=\phi-N^{r} \Gamma$ instead of $\phi$ and varies with respect to $\tilde{\phi}$ (Louko and Winters-Hilt 1996), but for our purposes it is sufficient to stick to the above form (33).

The model of spherical symmetric gravity can be embedded into a whole class of models usually referred to as "two-dimensional dilaton gravity theories". This terminology comes from effective two-dimensional theories (usually motivated by string theory) which contain in the gravitational sector a scalar field (the "dilaton") in addition to the two-dimensional metric (of which only the conformal factor is relevant). Interest in such models arose after Callan et al. (1992) studied one model in detail (now called the CGHS model), in which they addressed the issues of Hawking radiation and back reaction ${ }^{5}$. This was facilitated by the fact that this model is classically soluble even if another, conformally coupled, scalar field is included. The canonical formulation of this model can be found, e.g., in Louis-Martinez, Gegenberg, and Kunstatter (1994) and Demers and Kiefer (1996). The dilaton field is analogous to the field $R$ from above, while the conformal factor of the two-dimensional metric is analogous to $\Lambda$.

The dilaton model contains one non-trivial parameter, the constant $\lambda$ which has the dimension of an inverse length. The corresponding Hawking temperature and entropy are given by, respectively,

$$
T_{B H}=\frac{\hbar \lambda}{2 \pi k_{B}}, \quad S_{B H}=\frac{2 \pi k_{B} m}{\hbar \lambda} .
$$

Note that the temperature is here independent of the black hole mass $m$, and that therefore the entropy is linear in $m$. This is also the reason why some aspects of this models are unrealistic from the four-dimensional point of view.

Coming back to the spherically symmetric model, consider first the boundary conditions for $r \rightarrow \infty$. (If $r \in(-\infty, \infty)$, there are analogous conditions for $r \rightarrow-\infty$ which will be ignored here, see Kuchař (1994).) For $r \rightarrow \infty$ one has in particular

$$
\Lambda(r, t) \rightarrow 1+\frac{G m(t)}{r}, R(r, t) \rightarrow r, N \rightarrow N(t),
$$

\footnotetext{
${ }^{5}$ A detailed review of two-dimensional black holes is Strominger (1995).
} 
as well as

$$
P_{\Gamma}(r, t) \rightarrow q(t), \quad \phi(r, t) \rightarrow \phi(t) .
$$

From the variation with respect to $\Lambda$ one then finds the boundary term $G \int \mathrm{d} t N \delta m$. In order to avoid the unwanted conclusion $N=0$ (no evolution at infinity), one has to compensate this term in advance by adding the boundary term

$$
-G \int \mathrm{d} t N m
$$

to the classical action. Note that $m$ is just the ADM mass. The need to include such a boundary term was recognised by Regge and Teitelboim (1974). Similarly, for charged holes, one has to add the term

$$
-\int \mathrm{d} t \phi q
$$

to compensate for $\int \mathrm{d} t \phi \delta q$ which arises from varying $P_{\Gamma}$. If one wished instead to consider $q$ as a given, external parameter, this boundary term would be obsolete.

As long as restriction is made to the eternal hole, appropriate canonical transformations allow to simplify the classical constraint equations considerably (Kuchař 1994, Louko and Winters-Hilt 1996). One gets

$$
\left(\Lambda, P_{\Lambda} ; R, P_{R} ; \Gamma, P_{\Gamma}\right) \longrightarrow\left(\mathcal{M}, P_{\mathcal{M}} ; \mathcal{R}, P_{\mathcal{R}} ; Q, P_{Q}\right) .
$$

In particular,

$$
\begin{aligned}
\mathcal{M}(r, t) & =\frac{P_{\Gamma}^{2}+P_{\Lambda}^{2}}{2 R}+\frac{R}{2}\left(1-\frac{R^{\prime 2}}{\Lambda^{2}}\right) \stackrel{r \rightarrow \infty}{\longrightarrow} m(t) \\
Q(r, t) & =P_{\Gamma} \stackrel{r \rightarrow \infty}{\longrightarrow} q(t) .
\end{aligned}
$$

(I note that $\mathcal{R}=R$ and that the expression for $P_{\mathcal{R}}$ is somewhat lengthy and will not be given here.)

The new constraints, which are equivalent to the old ones, read

$$
\begin{gathered}
\mathcal{M}^{\prime}=0 \quad \Rightarrow \quad \mathcal{M}(r, t)=m(t), \\
Q^{\prime}=0 \quad \Rightarrow \quad Q(r, t)=q(t), \\
P_{\mathcal{R}}=0
\end{gathered}
$$

Note that $N(t)$ and $\phi(t)$ are prescribed functions that must not be varied; otherwise one would be led to the unwanted restriction that $m=0=q$. This can be remedied if the action is parametrised, bringing in new dynamical variables,

$$
\begin{aligned}
N(t) & =: \dot{\tau}(t), \\
\phi(t) & =: \dot{\lambda}(t) .
\end{aligned}
$$

Here, $\tau$ is the proper time that is measured with standard clocks at infinity, and $\lambda$ is the variable conjugate to charge; $\lambda$ is therefore connected with the elctromagnetic gauge parameter at the boundaries. In the canonical formalism 
one has to introduce momenta conjugate to these variables, which will be denoted $\pi_{\tau}$ and $\pi_{\lambda}$, respectively. This, in turn, requires the introduction of additional constraints linear in momenta,

$$
\begin{aligned}
& \mathcal{C}_{\tau}=\pi_{\tau}+G m \approx 0, \\
& \mathcal{C}_{\lambda}=\pi_{\lambda}+q \approx 0
\end{aligned}
$$

which have to be added to the action:

$$
\begin{aligned}
-G \int \mathrm{d} t m \dot{\tau} & \rightarrow \int \mathrm{d} t\left(\pi_{\tau} \dot{\tau}-N \mathcal{C}_{\tau}\right), \\
-\int \mathrm{d} t q \dot{\lambda} & \rightarrow \int \mathrm{d} t\left(\pi_{\lambda} \dot{\lambda}-\phi \mathcal{C}_{\lambda}\right) .
\end{aligned}
$$

The remaining constraints in this model are thus $(41)$ and $(43,44)$.

Quantisation proceeds then in the way sketched in Sect. 3.1 by acting with an operator version of the constraints on wave functionals $\Psi[\mathcal{R}(r) ; \tau, \lambda)$. Since (41) leads to $\delta \Psi / \delta \mathcal{R}=0$, one is left with a purely quantum mechanical wave function $\psi(\tau, \lambda)$. The implementation of the constraints $(43,44)$ then yields

$$
\begin{aligned}
& \frac{\hbar}{\mathrm{i}} \frac{\partial \psi}{\partial \tau}+m \psi=0, \\
& \frac{\hbar}{\mathrm{i}} \frac{\partial \psi}{\partial \lambda}+q \psi=0
\end{aligned}
$$

which can readily be solved to give

$$
\psi(\tau, \lambda)=\chi(m, q) \mathrm{e}^{-\mathrm{i}(m \tau+q \lambda) / \hbar}
$$

with an arbitrary function $\chi(m, q)$. Note that $m$ and $q$ are here considered as being fixed. The reason for this is that up to now we have restricted attention to one semiclassical component of the wave function only (eigenstates of mass and charge). Superpositions of states with different $m$ and $q$ can be made, and I shall make some remarks on this below.

If the hypersurface goes through the whole Kruskal diagramme of the eternal hole, only the boundary term at $r \rightarrow \infty$ (and an analogous one for $r \rightarrow-\infty$ ) contributes. Of particular interest in the black hole case, however, is the case where the surface originates at the bifurcation surface $(r \rightarrow 0)$ of past and future horizons. This makes sense since data on such a surface suffice to construct the whole right Kruskal wedge, which is all that is accessible to an observer in this region. Moreover, this mimics the situation where a black hole is formed by collapse, in which the regions $I I I$ and $I V$ of the Kruskal diagramme are absent.

What are the boundary conditions that are adopted at $r \rightarrow 0$ ? They are chosen in such a way that the classical solutions have a nondegenerate horizon and that the hypersurfaces begin at $r=0$ asymptotic to hypersurfaces of constant Killing time (Louko and Whiting 1995). In particular,

$$
\begin{aligned}
N(r, t) & =N_{1}(t) r+\mathcal{O}\left(r^{3}\right), \\
\Lambda(r, t) & =\Lambda_{0}(t)+\mathcal{O}\left(r^{2}\right), \\
R(r, t) & =R_{0}(t)+R_{2}(t) r^{2}+\mathcal{O}\left(r^{4}\right) .
\end{aligned}
$$


Variation leads, similarly to the situation at $r \rightarrow \infty$, to a boundary term at $r=0$ :

$$
-N_{1} R_{0}\left(G \Lambda_{0}\right)^{-1} \delta R_{0}
$$

If $N_{1} \neq 0$, this term must be subtracted $\left(N_{1}=0\right.$ corresponds to the case of extremal holes, $|q|=G m$, which is characterised by $\partial N / \partial r(r=0)=0$.) Introducing the notation $N_{0} \equiv N_{1} / \Lambda_{0}$, the boundary term to be added to the classical action reads

$$
(2 G)^{-1} \int \mathrm{d} t N_{0} R_{0}^{2}
$$

The quantity

$$
\alpha \equiv \int_{t_{1}}^{t} \mathrm{~d} t N_{0}(t)
$$

can be interpreted as a "rapidity" because it boosts the normal vector to the hypersurfaces $t=$ constant, $n^{a}$, in the way described by

$$
n^{a}\left(t_{1}\right) n_{a}(t)=-\cosh \alpha,
$$

see Hayward (1993). To avoid fixing $N_{0}$, one introduces an additional parametrisation (Brotz and Kiefer 1997)

$$
N_{0}(t)=\dot{\alpha}(t) .
$$

Similarly to $(45,46)$ above, one must replace in the action

$$
(2 G)^{-1} \int \mathrm{d} t R_{0}^{2} \dot{\alpha} \rightarrow \int \mathrm{d} t\left(\pi_{\alpha} \dot{\alpha}-N_{0} \mathcal{C}_{\alpha}\right),
$$

with the new constraint

$$
\mathcal{C}=\pi_{\alpha}-\frac{A}{8 \pi G} \approx 0
$$

where $A=4 \pi R_{0}^{2}$ is the surface of the bifurcation sphere. One notes that $\alpha$ and $A$ are canonically conjugate variables, see Carlip and Teitelboim (1995).

Quantisation then leads to (taking all constraints into account)

$$
\psi(\alpha, \tau, \lambda)=\chi(A, m, q) \exp \left(\frac{\mathrm{i}}{\hbar}\left[\frac{A}{8 \pi G} \alpha-m \tau-q \lambda\right]\right) .
$$

Since $A$ occurs in the state (58), one may suspect that also the entropy comes into play here, see (3). However, (58) is a pure quantum state, which possesses vanishing entropy, and $A$ is only part of its phase. The relation to entropy can only be achieved after an appropriate euclideanisation is performed, compare Sect. 2.3. This will be done below. (The wave function for a Reissner-Nordström hole, if an additional complex scalar field is coupled, can be found in Moniz (1997). In contrast to our model, his situation describes a dynamical evolution.)

The classical equations are found from (58) in the standard way by finding the extremum of the phase with respect to the parameters. For this to work, only two of the three parameters $A, m, q$ can be considered as independent. (I 
shall choose $m$ and $q$.) Differentiating the phase with respect to $m$ and setting the result to zero yields

$$
\alpha=8 \pi G\left(\frac{\partial A}{\partial m}\right)^{-1} \tau
$$

From Table 1 one recognises the occurrence of the surface gravity $\kappa$ on the righthand side of (59):

$$
\alpha=\kappa \tau,
$$

which is just the classical relation for the rapidity, see Brotz (1997). This is not surprising since it is known that boundary terms in the classical action are important in the derivation of the First Law of black hole mechanics (Wald 1997a). Generally, conjugate quantities in thermodynamics (extensive - intensive) correspond to conjugate variables in the Hamiltonian formalism.

Differentiating the phase of (58) with respect to $q$ and setting the result to zero yields

$$
\phi=\frac{\kappa}{8 \pi G} \frac{\partial A}{\partial q}=-\left.\frac{\partial m}{\partial q}\right|_{A}=-\frac{q}{R_{0}},
$$

another "thermodynamical" relation. ${ }^{6}$ This completes the solution of the eternal Reissner-Nordström hole.

I shall now turn to the more realistic case where an additional dynamical field is present. This can be used to "form" the black hole in the first place, and leads to the emergence of interesting features such as Hawking radiation. It also provides an interesting application of the semiclassical expansion presented in Sect. 3.1. I denote the scalar field by $f$, see e.g. Romano (1995), Demers and Kiefer (1996) and Kuchař et al. (1997) for details of the formalism.

At order $G^{0}$, the total wave functional is of the form

$$
\Psi \approx C^{g} \mathrm{e}^{\mathrm{i} S_{0}^{g} / \hbar} \bar{\chi},
$$

where $C^{g}$ and $S_{0}^{g}$ depend only on the gravitational (and electromagnetic) variables. These variables comprise the functions $\Gamma(r), R(r), \Lambda(r)$ as well as the boundary variables $\alpha, \tau, \lambda$. The functional $\bar{\chi}$ depends, in addition, on the scalar field $f$. The important point is that $\bar{\chi}$ obeys a functional Schrödinger equation with respect to the background found from $S_{0}^{g}$.

As in the general case, $S_{0}^{g}$ obeys the Hamilton-Jacobi equation for gravity (plus electromagnetic field). An explicit solution reads (Brotz and Kiefer 1997)

$$
\begin{aligned}
S_{0}^{g}= & \int_{0}^{\infty} \mathrm{d} r\left(q \Gamma+G^{-1} \Lambda F-G^{-1} \frac{R R^{\prime}}{2} \ln \frac{R^{\prime} / \Lambda+F / R}{R^{\prime} / \Lambda-F / R}\right) \\
& +\frac{A \alpha}{8 \pi G}-m \tau-q \lambda,
\end{aligned}
$$

where

$$
F=R \sqrt{\frac{R^{\prime 2}}{\Lambda^{2}}+\frac{2 M(r)}{R}-1}
$$

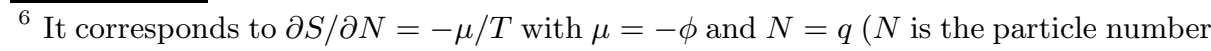
and $\mu$ the chemical potential.)
} 
and

$$
M(r)=m-\frac{q^{2}}{2 R(r)} .
$$

Note that $S_{0}^{g}$ depends parametrically on $m$ and $q$ which are just the mass and the charge of the hole, respectively. Expression (65) is nothing but the total energy of the hole. Inspection of (63) exhibits that the electromagnetic part in (62) from $S_{0}^{g}$ is given by

$$
\operatorname{expi}\left(\int_{0}^{\infty} \mathrm{d} r \Gamma-\lambda\right)
$$

This expression can be understood as follows. The electromagnetic potential (30) changes under a gauge transformations according to

$$
A \rightarrow \phi \mathrm{d} t+\Gamma \mathrm{d} r+\mathrm{d} \xi=(\phi+\dot{\xi}) \mathrm{d} t+\left(\Gamma+\xi^{\prime}\right) \mathrm{d} r .
$$

Therefore,

$$
\int_{0}^{\infty} \mathrm{d} r \Gamma(r) \rightarrow \int_{0}^{\infty} \mathrm{d} r \Gamma(r)+\xi(\infty)-\xi(0) .
$$

Now, $\xi(\infty)-\xi(0)$ may be absorbed into $\lambda$, since $\lambda$ itself was interpreted as the boundary gauge parameter.

Since the full theory is linear, one can perform arbitrary superpositions of states (62) with different values for $m$ and $q$. These describe situations where the hole has neither a definite charge nor a definite mass. However, such superpositions can only be distinguished from a corresponding mixture if one could "measure" the variables conjugate to $m$ and $q$, i.e. $\tau$ and $\lambda$. Otherwise, effective "superselection rules" would result, see Giulini, Kiefer, and Zeh (1995), and Chap. 6 of Giulini et al. (1996).

Another interesting situation is described by a superposition of the state (62) with its complex conjugate (this is possible since the full Wheeler-DeWitt equation is real). Such superpositions may follow in a natural way from appropriate boundary conditions (Hajicek 1992). It was shown in Demers and Kiefer (1996) that these superpositions (which can be heuristically interpreted as representing a superposition of a black hole with a white hole) become indistinguishable locally from a mixture after the irreversible interaction with the Hawking radiation is taken into account - a process known as decoherence (Giulini et al. 1996).

How can the Hawking radiation be found from a state such as (62)? This was clarified in Demers and Kiefer (1996) in the context of dilaton gravity (the extension to spherically symmetric gravity should be straightforward). One solves the functional Schrödinger equation obeyed by $\bar{\chi}$ in a background describing the collapse to a black hole. The initial state is taken to be a Gaussian (a "vacuum state"). During the evolution, this state remains a Gaussian, but with a different "width". This just expresses the fact, as mentioned in Sect. 2.2, that the notion of a vacuum becomes ambiguous in such a situation. Using the initial state as the 
reference vacuum state also at late times, the evolved state contains "particles" with respect to that vacuum. One has

$$
\langle\bar{\chi}|\hat{n}(k)| \bar{\chi}\rangle=\frac{1}{\exp \left(\frac{\hbar|k|}{k_{B} T_{B H}}\right)-1},
$$

where $\hat{n}$ denotes the "particle number operator" for the mode of wave number $k$ with respect to the original vacuum. Note that, although $\bar{\chi}$ is a pure quantum state, the expectation value (67) is a Planckian distribution with respect to the Hawking temperature $T_{B H}$. The difference of $\bar{\chi}$ to a genuine mixture will be noticed if other expectation values (of "higher order operators") are performed.

For the important case where the surfaces are fixed at the bifurcation sphere, it turns out that the field $f$ must vanish at this point for the state $\bar{\chi}$ to be normalisable. Thus, the bifurcation sphere acts like a "mirror" for this field. This is why the quantum state turns out to be a pure one. Other surfaces which penetrate the interior of the hole lead to a mixed state outside after the interior degrees of freedom are "traced out" (as in Israel 1976).

Can one go beyond the order of approximation (62)? This is in fact possible, but so far only in a formal way, without addressing in detail the issue of regularisation (Kiefer 1994). Still, however, qualitative features can be studied. At oder $G^{1}$, correction terms to the functional Schrödinger equation obeyed by $\bar{\chi}$ are obtained. Among these terms, there is an imaginary term, $\operatorname{im} H_{m}$, contributing to the effective matter Hamiltonian. In the case of collapse to a black hole, $\operatorname{Im} H_{m}<0$ (Kiefer, Müller, and Singh 1994). Since the following equation holds for the density matrix $\rho$,

$$
\frac{\mathrm{d}}{\mathrm{d} t}\left([\operatorname{Tr} \rho]^{2}-\operatorname{Tr} \rho^{2}\right)=4 \operatorname{Tr}\left(\left[\rho \operatorname{Tr} \rho-\rho^{2}\right] \operatorname{Im} H_{m}\right),
$$

one finds from $\operatorname{Im} H_{m}<0$ that the difference between $(\operatorname{Tr})^{2}$ and $\operatorname{Tr} \rho^{2}$ decreases, corresponding to an increase in "purity" for the quantum state. Whether this may indicate a quantum gravitational "recovery of information" from the hole can of course only be judged from the full, as yet elusive, theory. This result at least demonstrates what kind of effects one might expect to see in higher orders of the semiclassical approximation.

At order $G^{1}$, also back reaction terms from the matter fields (here from the $f$-field) onto the gravitational background are found (Kiefer 1994). These can be evaluated only in special cases, for example in the toy model of a 2+1dimensional black hole coupled to a conformal scalar field (Brotz 1998).

An interesting point is of course whether there are situations where the semiclassical approximation breaks down in the first place. This would mean that quantum gravity effects can become important below the Planck scale. KeskiVakkuri et al. (1995), for example, arrived at the conclusion that the semiclassical approximation breaks down at the black hole horizon, in the sense that tiny fluctuations of the black hole mass may produce an immense change in the matter state. The physical implications of this result are not yet fully clear. It can also 
not be excluded that anomalies in quantum gravity spoil the above semiclassical limit and demand for an explicit modification of the constraints, see e.g. Cangemi, Jackiw, and Zwiebach (1996).

I emphasised above that there is not yet any connection with a notion of entropy for the pure quantum state (58). This can be established after some "euclideanisation" is performed, see the discussion in Sect. 2.3. How does this work? From (55) it is clear that the rapidity $\alpha$ is connected with the lapse function. Therefore, going to the euclidean regime means both $\tau \rightarrow-\mathrm{i} \beta \hbar$, see (14), and $\alpha \rightarrow-\mathrm{i} \alpha_{E}$. Regularity of the line element then demands that $\alpha_{E}=2 \pi$ (Brotz and Kiefer 1997). Consequently, the euclidean version of the quantum state (62) contains the term

$$
\exp \left(-\beta m+\frac{A}{4 \hbar G}\right)
$$

There is in addition the euclideanised version of the integral in (63) and the term containing $\lambda \rightarrow \lambda_{E}=-\mathrm{i} \hbar \beta \phi$.

This does of course not yet yield a partition sum. However, after the whole semiclassical part is evaluated at the classical value for the Hamilton-Jacobi functional and a trace is performed, one finds by applying (17) that the second term in (69) is just the Bekenstein-Hawking entropy (3). Alternatively, one can interpret (69) as directly giving the enhancement factor for the rate of black hole pair creation relative to ordinary pair creation. Here my focus was just to show how the expression for $S_{B H}$ emerges in the canonical formalism. ${ }^{7}$

Consider now the case of an extremal hole, where $|q|=G m$. As can be immediately inferred from the discussion after (52), there is no surface term to consider, since $N_{0}=0$. Thus, $\alpha=0$, and there is no $A$-term in (58). This would also mean that the entropy is zero. Recalling our discussion in Sect. 2.1, this shows that Planck's version of the Third Law is fulfilled. This result was also found in a variety of other approaches, see the references in Brotz and Kiefer (1997). It is not fulfilled in string theory, where $S_{\text {extreme }}=A / 4 \hbar G$, see Sect. 4 . It is also not fulfilled for the extreme (Kerr) black hole which occurs in the transition from the disk of dust solution to the rotating black hole solution, see Neugebauer's contribution to this volume.

The above derivation of entropy via boundary terms suggests the following natural interpretation in terms of "missing information". For surfaces which in the classical spacetime correspond to slices through the full Kruskal diagramme, this "information" is maximal in the sense that one can recover the full spacetime from data on this surface. Since no boundary (except at infinity) is present, the entropy is zero. For slices that start at the bifurcation sphere, this information is less than maximal for Schwarzschild black holes and for non-extreme ReissnerNordström black holes. They are therefore attributed the entropy $A / 4 \hbar G$. In contrast, the maximum information (for the full spacetime up to the Cauchy

\footnotetext{
${ }^{7}$ Due to Smarr's formula, (69) is consistent with (21).
} 
horizon) is already available for such slices in the extreme case, as can be easily recognised from the corresponding Penrose diagramme. Extreme holes are therefore attributed a vanishing entropy. A somewhat related interpretation was given in the path integral framework by Martinez (1995). An interesting point was raised by Ghosh and Mitra (1997) who argued that $S_{\text {extreme }} \neq 0$ follows from extremisation after quantisation, while $S_{\text {extreme }}=0$ holds for extremisation before quantisation.

Can the quantisation of mass (or area), as described by (24), be found within the canonical formalism? This is, unfortunately, an open issue. One can, for example, postulate Bohr-Sommerfeld type of quantisation rules in the euclidean theory (Kastrup 1996). This would lead to

$$
n h=\oint \pi_{\alpha} \mathrm{d} \alpha=\int_{0}^{2 \pi} \frac{A}{8 \pi G} \mathrm{~d} \alpha=\frac{A}{4 G} .
$$

This is similar to (24), albeit with a different numerical factor. Whether a similar result can be found in the physically relevant lorentzian theory remains open.

Other interesting developments can only be mentioned here. Carlip (1997) was able to give a statistical mechanical origin for the black hole entropy in the case of a $2+1$ dimensional black hole. There it results from "would-be-gauge" degrees of freedom becoming dynamical at the horizon. Using the loop approach to canonical quantum gravity, Rovelli (1996) found that $S_{B H} \propto A$, although with a numerical coefficient different from (3).

To summarise, canonical quantum gravity can offer the tool to understand quantum features of black holes such as entropy and Hawking radiation. Still, however, the main problems are not yet solved: Can the Bekenstein-Hawking entropy for four-dimensional black holes be derived by counting appropriate degrees of freedom? What is the final evolution of a black hole, after the semiclassical approximation breaks down?

\section{Further Developments}

In Sect. 3 I discussed canonical quantum gravity as a possible framework to understand black holes. A different approach to quantum gravity is superstring theory. It necessarily contains gravity and gauge theories, and must implement supersymmetry for reasons of consistency.

Like canonical quantum gravity, string theory follows through the quantisation of a classical theory (a propagating string in some background spacetime), but is itself interpreted in a drastically different way: It is supposed to give a fundamental theory where all interactions including gravity are unified in a quantum framework. The background spacetime used in the construction of the theory plays only an auxiliary role. Like canonical quantum gravity, string theory suffers from the "problem of time", although this is not always stated clearly. The notion of spacetime again emerges only in an appropriate semiclassical limit. (The role of the semiclassical expansion parameter is here played by 
the string length, see below). An important fact in string theory is that consistency conditions (the absence of a Weyl anomaly) severely restricts the number of dimensions of this semiclassical spacetime, e.g. to $D=10$ for the superstring. This, then, enforces the implementation of an appropriate mechanism to encurl the superfluous dimensions in a Kaluza-Klein type manner to avoid contradiction with observation. Whether the level of canonical quantum gravity, as discussed in Sect. 3, follows from string theory in an appropriate limit is not yet clear. It must, however, lead to some quantum gravitational corrections to the ordinary functional Schrödinger equation, and may thus lead to the possibility both to test the theory and to discriminate it from competitors like the approach presented in Sect. 3.

A detailed introduction into string theory can be found, for example, in Polchinski (1994, 1996), and the references therein. Here I only want to briefly sketch some intriguing recent developments aiming at a derivation of the black hole entropy (3) by counting quantum states, see Horowitz (1997) for a review. String theory contains two important parameters: The string length $l_{S}$ and the string coupling $g_{S}^{2} \equiv \exp (2 \varphi)$. Here, $\varphi$ denotes the dilaton field which appears in the two-dimensional string action. It gives rise to the string coupling, since $g_{S}^{2}$ appears as a "gravitational constant" in the effective action (arising in the semiclassical approximation to lowest order in $l_{S}$ ) for the background spacetime and background fields. The Planck length, $l_{P}$, then appears as a derived quantity,

$$
l_{P} \propto g_{S} l_{S},
$$

and similar relations follow for other "coupling constants". It is important to note that the semiclassical approximation, and with it the notion of a spacetime metric, breaks down for curvatures bigger than $l_{S}^{-2}$.

How does the entropy of a black hole come into play? First, assigning an entropy to an excited string state by counting its degeneracy, it turns out that this entropy is (for high excitations) proportional to the energy (mass) of that state and not to the mass squared. It would thus seem as if a string had not enough states to yield the entropy of a black hole. The crucial point, however, is that the Planck length, and therefore the gravitational constant, depends on the string coupling, see (71). Thus, if $g_{S}$ is increased, $G m$ is increased, too, and a black hole is formed at some stage (Horowitz 1997). Comparing, then, the black hole mass with the string mass at $l_{S}=R_{0}$ ( $R_{0}$ is the Schwarzschild radius), it turns out that the black hole entropy becomes proportional to the string entropy. A string may thus possess enough states to give the Bekenstein-Hawking entropy.

For a quantitative comparison, one must give a precise calculation. It is most straightforward in this respect to first consider states which obey a relation similar to $q=G m$ in the Reissner-Nordström case (although with generalised charges). Such states are called BPS states. At weak coupling $\left(g_{S} \ll 1\right)$, one has bound states of so-called D-branes (Polchinski 1996) in flat space, and the number of these states can be counted. D-branes are dynamical objects of various dimensions, which are a necessary ingredient of string theory. As the coupling increases, the BPS-relation between mass and charges is preserved, and 
the number of states remains unchanged. For high coupling $\left(g_{S} \gg 1\right)$, one thus obtains an extremal black hole with the same number of states. Surprisingly, its Bekenstein-Hawking entropy exactly coincides with the entropy of the Dbranes in the flat space description (Strominger and Vafa 1996). The original calculation was for five-dimensional black holes and then generalised to fourdimensional holes. One may thus interpret the D-branes as giving the desired microscopic description for the black hole entropy. Since it turns out in this approach that $S_{\text {extreme }}=A / 4 \hbar G \neq 0$, string theory leads to a different result than the canonical treatment presented in Sect. 3.2.

The calculations have been extended to the case of near-extremal black holes which, in contrast to the extremal ones, exhibit Hawking radiation (here interpreted as the emission of closed strings from D-branes). It could be shown that even the rate of Hawking radiation agrees with the decay amplitude for the corresponding D-brane configuration (see e.g. Das 1997). Since all string calculations preserve unitarity, it seems that there is no violation of unitarity also in the black hole radiation. Consequently, there would be no "loss of information". Of course, to get a non-vanishing entropy in the first place, some coarse-graining must be involved, and the process of decoherence will again play a crucial role (Myers 1997). There will thus only be the apparent non-unitarity connected with the neglect of degrees of freedom be present - the total system evolves unitarily (Giulini et al. 1996).

Whether the above string result also holds for general black holes, i.e. far away from extremality (such as for the Schwarzschild black hole), is not yet clear. It must also be emphasised that all results are obtained in lowest order of $l_{S}$, i.e. in the lowest order of the semiclassical approximation where a background structure is available. The full, non-perturbative, evolution of a black hole therefore still remains mysterious.

In the semiclassical approximation to canonical quantum gravity, as presented in Sect. 3, a crucial role for the interpretation of entropy is played by the presence of boundary conditions at the bifurcation sphere (where the two horizons in the Kruskal diagramme meet). This, however, cannot be extended to the full theory in a straightforward manner. The main reason is that the horizon of a black hole is a classical concept. As I emphasised in Sect. 3.1, the canonical theory does not possess any notion of spacetime at the fundamental level, in the same way as ordinary quantum theory does not possess any notion of particle trajectories in the full theory. A horizon, however, is a genuine spacetime concept. Therefore, the results presented in Sect. 3.2 only hold as far as a notion of spacetime can be applied at least in some approximation.

That the concept of an event horizon is a classical artifact, becomes especially obvious in quantum cosmology (Zeh 1992). Consider, for example, the case of a Friedmann universe that classically recollapses. Since the entropy content of the present universe is far from maximal, it must have been very tiny at the big bang - the big bang was extremely smooth (which is why one would not expect to find many primordial black holes). This led Penrose (1979) to the formulation of his Weyl tensor hypothesis that the universe is homogeneous at 
the big bang, but not at the big crunch. In quantum gravity, however, there is no external time parameter which could possibly distinguish between big bang and big crunch. If entropy is small near the big bang, it must also be small near the big crunch, since both regions correspond to the same region of the quantum gravitational configuration space. The consequences of this fact for the arrow of time and for black holes were investigated in Kiefer and Zeh (1995). Entropy is always growing with increasing size of the Universe, leading to a (formal) reversal of the arrow of time near the classical turning point. The same boundary condition of low entropy at small size necessarily leads to the fact that neither an event horizon nor a singularity (naked or hidden) forms for a black hole. Cosmic censorship would thus be automatically implemented. Although still speculative, this scenario at least demonstrates what qualitatively new features emerge from quantum gravity if one leaves the semiclassical sector.

Acknowledgements. I am grateful to Thorsten Brotz, Valeri Frolov, Domenico Giulini, and H.-Dieter Zeh for a critical reading of this manuscript.

\section{References}

Ashtekar, A. (1997): Polymer geometry at Planck scale and quantum Einstein equations. In General Relativity and Gravitation, edited by M. Francaviglia, G. Longhi, L. Lusanna, and E. Sorace (World Scientific, Singapore), p. 3-29

Barvinsky, A.O. (1993): Operator ordering in theories subject to constraints of the gravitational type. Class. Quantum Grav. 10, 1985-1999

Barvinsky, A.O., Frolov, V.P., Zelnikov, A.I. (1995): The wave function of a black hole and the dynamical origin of entropy. Phys. Rev. D 51, 1741-1763

Barvinsky, A.O., Krykhtin, V. (1993): Dirac and BFV quantization methods in the 1-loop approximation: closure of the quantum constraint algebra and the conserved inner product. Class. Quantum Grav. 10, 1957-1984

Bekenstein, J.D. (1980): Black-hole thermodynamics. Physics Today (January), 24-31

Bekenstein, J.D. (1997): Quantum black holes as atoms. Report gr-qc/9710076, to appear in the Proccedings of the VIII Marcel Grossmann Meeting, ed. by R. Ruffini and T. Piran (World Scientific, Singapore)

Bell, J.S., Leinaas, J.M. (1987): The Unruh effect and quantum fluctuations of electrons in storage rings. Nucl. Phys. B 284, 488-508

Bertoni, C., Finelli, F., Venturi, G. (1996): The Born-Oppenheimer approach to the matter-gravity system and unitarity. Class. Quantum Grav. 13, 2375-2383

Birrell, N.D., Davies, P.C.W. (1982): Quantum fields in curved space (Cambridge University Press, Cambridge)

Bombelli, L., Koul, R.K., Lee, J., Sorkin, R.D. (1986): Quantum source of entropy for black holes. Phys. Rev. D 34, 373-383

Bousso, R., Hawking, S.W. (1997): Black holes in inflation. Nucl. Phys. B (Proc. Suppl.) 57, 201-205

Braden, H.W., Brown, J.D., Whiting, B.F., York, J.W., Jr. (1990): Charged black hole in a grand canonical ensemble. Phys. Rev. D 42, 3376-3385

Brotz, T. (1998): Quantization of Black Holes in the Wheeler-DeWitt Approach. Phys. Rev. D 57, 2349-2362 
Brotz, T., Kiefer, C. (1997): Semiclassical black hole states and entropy. Phys. Rev. D 55, 2186-2191

Brown, J.D. (1995): Black hole entropy and the Hamiltonian formulation of diffeomorphism invariant theories. Phys. Rev. D 52, 7011-7026

Brown, J.D., York, J.W., Jr. (1993): Microcanonical functional integral for the gravitational field. Phys. Rev. D 47, 1420-1431

Callan, C.G., Jr., Giddings, S.B., Harvey, J.A., Strominger, A. (1992): Evanescent black holes. Phys. Rev. D 45, R1005-R1009

Cangemi, D., Jackiw, R., Zwiebach, B. (1996): Physical States in Matter-Coupled Dilaton Gravity. Ann. Phys. (N.Y.) 245, 408-444

Carlip, C. (1997): Statistical mechanics and black hole thermodynamics. Nucl. Phys. B (Proc. Suppl.) 57, 8-12

Carlip, C., Teitelboim, C. (1995): The off-shell black hole. Class. Quantum Grav. 12, 1699-1704

Cavaglià, M., de Alfaro, V., Filippov, A.T. (1996): Quantization of the Schwarzschild Black Hole. Int. J. Mod. Phys. D 5, 227-250

Choptuik, M.W. (1993): Universality and Scaling in Gravitational Collapse of a Massless Scalar Field. Phys. Rev. Lett. 70, 9-12

Das, S.R. (1997): D-brane decay and Hawking Radiation. Report hep-th/9709206

Davies, P.C.W. (1977): The thermodynamic theory of black holes. Proc. R. Soc. Lond. A 353, 499-521

Demers, J.-G., Kiefer, C. (1996): Decoherence of black holes by Hawking radiation. Phys. Rev. D 53, 7050-7061

Eberlein, C. (1990): Theory of quantum radiation observed as sonoluminescence. Phys. Rev. A 53, 2772-2787

Ehlers, J., Friedrich, H. (1994), eds.: Canonical Gravity: From Classical to Quantum (Springer, Berlin)

Feynman, R.P., Hibbs, A.R. (1965): Quantum Mechanics and Path Integrals (McGrawHill, New York)

Frolov, V.P., Fursaev, D.V. (1998): Thermal Fields, Entropy, and Black Holes. Report hep-th/9802010

Frolov, V.P., Fursaev, D.V., Zelnikov, A.I. (1997): Statistical origin of black hole entropy in induced gravity. Nucl. Phys. B 486, 339-352

Frolov, V., Novikov, I. (1993): Dynamical origin of the entropy of a black hole. Phys. Rev. D 48, 4545-4551

Frolov, V., Page, D.N. (1993): Proof of the Generalized Second Law for Quasistationary Semiclassical Black Holes. Phys. Rev. Lett. 71, 3902-3905

Ghosh, A., Mitra, P. (1997): Understanding the Area Proposal for Extremal Black Hole Entropy. Phys. Rev. Lett. 78, 1858-1860

Gibbons, G.W., Hawking, S.W. (1977): Action integrals and partition functions in quantum gravity. Phys. Rev. D 15, 2752-2756

Gibbons, G.W., Perry, M.J. (1978): Black holes and thermal Green functions. Proc. R. Soc. Lond. A 358, 467-494

Giddings, S.B. (1994): Quantum Mechanics of Black Holes. Report hep-th/9412138

Giulini, D., Joos, E., Kiefer, C., Kupsch, J., Stamatescu, I.-O., Zeh, H.D. (1996): Decoherence and the Appearance of a Classical World in Quantum Theory (Springer, Berlin)

Giulini, D., Kiefer, C., Zeh, H.D. (1995): Symmetries, superselection rules, and decoherence. Phys. Lett. 199A, 291-298 
Hajicek, P. (1992): Quantum Mechanics of Gravitational Collapse. Commun. Math. Phys. 150, 545-559

Halliwell, J.J. (1991): Introductory lectures on quantum cosmology. In Quantum cosmology and baby universes, ed. by S. Coleman, J.B. Hartle, T. Piran, and S. Weinberg (World Scientific, Singapore), p. 159-243

Hartle, J.B., Hawking, S.W. (1976): Path-integral derivation of black-hole radiance. Phys. Rev. D 13, 2188-2203

Hartle, J.B., Hawking, S.W. (1983): Wave function of the Universe. Phys. Rev. D 28, 2960-2975

Hawking, S.W. (1975): Particle Creation by Black Holes. Commun. Math. Phys. 43, 199-220

Hawking, S.W. (1979): The path-integral approach to quantum gravity. In General Relativity, ed. by S.W. Hawking and W. Israel (Cambridge University Press, Cambridge), p. 746-789

Hawking, S.W., Penrose, R. (1996): The Nature of Space and Time (Princeton University Press, Princeton)

Hawkins, M.R.S. (1993): Gravitational microlensing, quasar variability and missing matter. Nature (London) 366, 242-245

Hayward, G. (1993): Gravitational action for spacetimes with nonsmooth boundaries. Phys. Rev. D 47, 3275-3280

Heusler, M. (1996): Black Hole Uniqueness Theorems (Cambridge University Press, Cambridge)

Horowitz, G.T. (1997): Quantum States of Black Holes. Report gr-qc/9704072

Isham, C.J. (1997): Structural issues in quantum gravity. In General Relativity and Gravitation, ed. by M. Francaviglia, G. Longhi, L. Lusanna, and E. Sorace (World Scientific, Singapore), p. 167-209

Israel, W. (1976): Thermo-field dynamics of black holes. Phys. Lett. 57A, 107-110

Israel, W. (1986): Third Law of Black-Hole Dynamics: A Formulation and Proof. Phys. Rev. Lett. 57, 397-399

Iyer, V., Wald, R.M. (1994): Some properties of the Noether charge and a proposal for dynamical black hole entropy. Phys. Rev. D 50, 846-864

Iyer, V., Wald, R.M. (1995): Comparison of the Noether charge and Euclidean methods for computing the entropy of stationary black holes. Phys. Rev. D 52, 4430-4439

Jacobson, T. (1995): Thermodynamics of Spacetime: The Einstein Equation of State. Phys. Rev. Lett 75, 1260-1263

Kastrup, H.A. (1996): The quantum levels of isolated spherically symmetric gravitational systems. Phys. Lett. B bf 385, 75-80

Kastrup, H.A., Thiemann, T. (1994): Spherically symmetric gravity as a completely integrable system. Nucl. Phys. B 425, 665-686

Keski-Vakkuri, E., Lifschytz, G., Mathur, S.D., Ortiz, M.E. (1995): Breakdown of the semiclassical approximation at the black hole horizon. Phys. Rev. D 51, 1764-1780

Kiefer, C. (1994): The semiclassical approximation to quantum gravity. In: Ehlers and Friedrich (1994), p. 170-212

Kiefer, C. (1997a): Quanteneigenschaften Schwarzer Löcher. Physik in unserer Zeit 28, $22-30$

Kiefer, C. (1997b): Does time exist at the most fundamental level? In: Time, Temporality, Now, ed. by H. Atmanspacher and E. Ruhnau (Springer, Berlin), p. 227-240

Kiefer, C., Müller, R., Singh, T.P. (1994): Quantum Gravity and Non-Unitarity in Black Hole Evaporation. Mod. Phys. Lett. A 9, 2661-2669 
Kiefer, C., Zeh, H.D. (1995): Arrow of time in a recollapsing quantum universe. Phys. Rev. D 51, 4145-4153

Kim, S.P. (1997): Problem of unitarity and quantum corrections in semiclassical quantum gravity. Phys. Rev. D 55, 7511-7517

Kuchař, K.V. (1994): Geometrodynamics of Schwarzschild black holes. Phys. Rev. D 50, 3961-3981

Kuchař, K.V., Romano, J.D., Varadarajan, M. (1997): Dirac constraint quantization of a dilatonic model of gravitational collapse. Phys. Rev. D 55, 795-808

Louis-Martinez, D., Gegenberg, J., Kunstatter, G. (1994): Exact Dirac quantization of all 2D dilaton gravity theories. Phys. Lett. B 321, 193-198

Louko, J., Whiting, B.F. (1995): Hamiltonian thermodynamics of the Schwarzschild black hole. Phys. Rev. D 51, 5583-5599

Louko, J., Winters-Hilt, S.N. (1996): Hamiltonian thermodynamics of the ReissnerNordström-anti-de Sitter black hole. Phys. Rev. D 54, 2647-2663

Martinez, E.A. (1995): Microcanonical functional integral and entropy for eternal black holes. Phys. Rev. D 51, 5732-5741

Moniz, P.V. (1997): Wave function for the Reissner-Nordström black hole. Mod. Phys. Lett. A 12, 1491-1505

Myers, R. (1997): Pure states don't wear black. Gen. Rel. Grav. 29, 1217-1222

Page, D.N., Hawking, S.W. (1976): Gamma rays from primordial black holes. Astrophys. Journ. 206, 1-7

Parentani, R., Massar, S. (1997): Schwinger mechanism, Unruh effect, and production of accelerated black holes. Phys. Rev. D 55, 3603-3613

Penrose, R. (1979): Singularities and Time-Asymmetry. In General Relativity, ed. by S.W. Hawking and W. Israel (Cambridge University Press, Cambridge), p. 581-638

Polchinski, J. (1994): What is string theory? Report hep-th/9411028

Polchinski, J. (1996): TASI Lectures on D-branes. Report hep-th/9611050

Regge, T., Teitelboim, C. (1974): Role of Surface Integrals in the Hamiltonian Formulation of General Relativity. Ann. Phys. (N.Y.) 88, 286-318

Romano, J.D. (1995): Spherical Symmetric Scalar Field Collapse: An example of the Spacetime Problem of Time. Report gr-qc/9501015

Rovelli, C. (1996): Black Hole Entropy from Loop Quantum Gravity. Phys. Rev. Lett. 77, 3288-3291

Srednicki, M. (1993): Entropy and Area. Phys. Rev. Lett. 71, 666-669

Straumann, N. (1986): Thermodynamik (Springer, Berlin), p. 100

Strominger, A. (1995): Les Houches Lectures on Black Holes. Report hep-th/9501071

Strominger, A., Vafa, C. (1996): Microscopic origin of the Bekenstein-Hawking entropy. Phys. Lett. B 379, 99-104

't Hooft, G. (1996): The scattering matrix approach to the quantum black hole: an overview. Int. J. Mod. Phys. A 11, 4623-4688

Thiemann, T., Kastrup, H.A. (1993): Canonical quantization of spherically symmetric gravity in Ashtekar's self-dual representation. Nucl. Phys. B 399, 211-258

Unruh, W.G. (1976): Notes on black-hole evaporation. Phys. Rev. D 14, 870-892

Unruh, W.G., Wald, R.M. (1982): Acceleration radiation and the generalized second law of thermodynamics. Phys. Rev. D 25, 942-958

Wald, R.M. (1994): Quantum Field Theory in Curved Spacetime and Black Hole Thermodynamics (University of Chicago Press, Chicago)

Wald, R.M. (1997a): Black Holes and Thermodynamics. Report gr-qc/9702022 
Wald, R.M. (1997b): The "Nernst Theorem" and Black Hole Thermodynamics. Phys. Rev. D 56, 6467-6474

Wilks, J. (1961): The third law of thermodynamics (Oxford University Press, Oxford) Wright, E.L. (1996): On the density of primordial black holes in the galactic halo. Astrophys. Journ. 459, 487-490

York, J.W., Jr. (1986): Black-hole thermodynamics and the Euclidean Einstein action. Phys. Rev. D 33, 2092-2099

York, J.W., Jr. (1991): Black holes and Partition Functions. In Conceptual Problems of Quantum Gravity, ed. by A. Ashtekar and J. Stachel (Birkhäuser, Boston), p. 573596

Zeh, H.D. (1992): The Physical Basis of the Direction of Time (Springer, Berlin)

Zurek, W.H., Thorne, K.S. (1985): Statistical Mechanical Origin of the Entropy of a Rotating, Charged Black Hole. Phys. Rev. Lett. 54, 2171-2175 